\title{
Sleep-Related Leg Movement Disorders
}

\section{Samavedam A. Krishna}

See end of article for authors affiliations

Indian J Sleep Med 2006; 1.1, 12-18

Address for correspondence: Samavedam A. Krishna, M.D. Diplomate, American Boards of Internal Medicine and Sleep Medicine, Mount Vernon, O hio, USA

\begin{abstract}
Restless-legs syndrome and periodic leg movements in sleep are sleep-related limb movement disorders that often disrupt nocturnal sleep and cause excessive daytime sleepiness. This article reviews the recent literature regarding epidemiology, etiology, pathogenesis, clinical features, differential diagnosis and management.
\end{abstract}

\section{Introduction}

D

aytime sleepiness, fatigue, tiredness and restless night sleep are some of the most frequent symptoms that patients report to their primary care physicians and somnologists. Among the most important reasons for these presenting symptoms, in addition to sleep-disordered breathing, are the sleeprelated leg movement disorders restless-legs syndrome (RLS) and periodic leg movements in sleep (PLMS). While the majority of patients or their relatives can recognize, even at a non-technical level, that snoring and apneic episodes in sleep are not normal and are probably contributing to their sleep-related symptoms, relatively few patients are able to recognize the association between movement disorders and sleep. RLS remains characterized, despite advances in understanding of its etiopathogenesis and therapy for it, by the paradox of poor self-recognition of symptoms, misdiagnosis despite easy-to-apply clinical diagnostic criteria and inappropriate treatment despite availability of effective treatments.

RLS and PLM S may occur separately but often cooccur to causesignificant sleep disruption and consequent daytime symptoms. RLS was described about 60 years ago [1] but precise, universally accepted definition of the syndrome has only become available in the last 10 years $[2,3]$. Along with this has come a tremendous growth in identification of the disorders in the clinical setting and realization of their adverse effects on sleep.

\section{Epidemiology \& etiopathogenesis}

RLS is fairly common in Caucasians, with a prevalence rate of $5-15 \%$ in the general population as per several estimates in Europe and $\mathrm{N}$ orth America[4, 5, 6, 7]. It is more common in women. There appear to be two patterns of occurrence - early onset (i.e. well before the age of 45 years) and late onset. Early onset RLS is more often associated with presence of family history of the problem (in first and second degree relatives) and very gradual progression to bothersome symptoms over a period of several decades. The course of mild RLS is often marked by extended symptom-free periods. Late onset RLS is more often secondary to other medical problems and tends to result in bothersome and frequent symptoms more rapidly than the early onset form. Secondary RLS also tends to improve as the underlying condition is corrected or controlled. As the frequency of RLS increases in terms of the numbers of days per week with symptoms and intensity of discomfort in the legs, many patients also experience similar symptoms in the upper extremities.

Even though familial association has been documented, with concordance shown in monozygotic twins [8] and in large kindreds, no clear pattern of inheritancehas emerged. The chromosomal loci to which RLS has been localized has been different in three out of four such studies of inheritance patterns of RLS $[9,10$, $11]$. 
Prevalence rates for RLS are even higher in certain otherwise-normal population subsets such as pregnant women and blood donors [12]. The prevalence of SRLMD and associated symptoms is also far greater than normal in disorders such as iron deficiency anemia [13], chronic renal failure [14], B12 and/or folic acid deficiency and probably diabetes mellitus.

Interestingly, sparse epidemiologic data from India $[15,16]$, Singapore [17] and J apan [18] suggest far lower prevalence rates in both specific population subsets such as uremic or Parkinsonian individuals and controls. $\mathrm{H}$ owever, cross-sectional studies of adequate size in these populations haven't been reported to date.

Rapid and complete relief of RLS and PLMS by administration of even small doses of dopaminergic medications points to the role of dysfunction of dopaminergic neurotransmission in SRLMD. Further supporting this idea, dopamine receptor antagonists such as metoclopramide cause or worsen RLS. Some (though not all) neuroimaging studies have shown reduced dopamine 2 receptor binding in thestriatum and others have shown circadian variation in dopamine $D 1$ receptor binding $[19,20]$. I ron deficiency was initially thought to produce SRLM D by adversely affecting tyrosine hydroxylase, the rate limiting enzyme needed for the synthesis of dopamine from L-D OPA. RLS may occur in the presence of even low-grade iron deficiency without overt anemia and correction of iron deficiency has been shown to improve SRLM D in overt iron deficiency and end-stage renal failure states [21, 22]. H owever, no actual deficiency of dopamine in the cerebro-spinal fluid (CSF) has been demonstrated in iron deficiency. Instead, based on the occurrence of RLS in hemochromatosis [23], it appears that dysregulation of iron in the central nervous system is the actual mechanism underlying SRLMD. Evidence for iron dysregulation includes reduced ferritin and increased transferrin levels in neuromelanin cells and distribution of the L-ferritin subunit of ferritin to surrounding microglia that may result in localized abnormalities of dopamine, dopamine receptor expression and dopamine receptor binding [24, 25].

In addition to iron deficiency, diabetes mellitus and B12 / folic acid deficiency may also be associated with SRLM D though studies in this regard have been inconclusive so far $[26,27]$. Small doses of dopamine receptor agonists seem to behighly effectivein controlling SRLM D in patients who turn out to have diabetes, prediabetes or B12 / folic acid deficiency. Reduced striatal dopamine $D 2$ receptor availability associated with increased appetite and obesity has been demonstrated by neuroimaging and central dopaminergic tone appears to be inversely related to development of obesity, insulin resistance and diabetes mellitus [28]. B12 / folic acid deficiency may adversely affect dopamine production [29].

Thus, dysfunction of dopaminergic neurotransmission in some dopaminergic pathways in the CNS appears to be the final common pathway by which the various secondary conditions (iron deficiency and dysregulation, diabetes mellitus and B12 / folic acid deficiency) produce SRLM D.

\section{Clinical features \& diagnostic criteria Restless Legs Syndrome}

There are four essential criteria for the diagnosis of RLS, as per the International RLS Study G roup and the N IH consensus panel of 2003.

- There is an urge to move the legs accompanied by or caused by unpleasant sensations in the legs. Patients describe many variants of the uncomfortable sensations, including "aching," "painful," "creeping," "crawling," and "tightness." The sensations are usually felt deep in the limb. As SRLMD worsen, many patients note similar sensations in the upper extremities also.

- The urge to move the legs and associated sensations occur during inactivity or rest. The duration of inactivity or rest needed to trigger RLS varies and some people only experience RLS during meetings or long car rides later in the day.

- The urge to move the legs and the associated sensations are relieved by movement or application of a counter-stimulus, at least for as long as the movement or counter-stimulus is maintained. Examples include rhythmic movement of the legs, walking, rubbing one foot against the other leg, bathing and walking on a cool surface. "Tossing and turning" to get comfortable in bed at night is also described by many patients. $O$ ften the affected person is only minimally aware of the movement.

- The urge to move the legs and the accompanying sensations either occur only in the evening or night or worsen in the evening or night. As RLS worsens, the urge to move and the accompanying sensations

Indian Journal of Sleep M edicine (IJSM ), Vol. 1, N o. 1, 2006 
may develop much earlier in the day. In such cases, previous circadian variation in symptoms is necessary.

Three factors that support but are not essential to the diagnosis of RLS have been described by the International RLS Study Group and N IH Consensus Panel:

- Positive family history of RLS in first or second degree relatives.

- Response to dopaminergic medication in low doses.

- Occurrence of periodic leg movements (both during wakeful rest and in sleep).

In children, a description of the leg discomfort in the child's own words is necessary in addition to the above four primary diagnostic criteria, in order to make the diagnosis of RLS. If the child is unable to provide such a verbal description of leg discomfort, the four primary diagnostic criteria and at least two of the three supportive criteria must be met in order to clinch the diagnosis. If first degree relatives of the child have RLS and the four primary diagnostic criteria can't be met or can only be inferred by observation, only "probable" RLS can be concluded. RLS may be "possible" if the child has periodic leg movements in sleep and a family history of RLS. Likewise, in cognitively impaired adults, the primary diagnostic criteria may be inferred by observation though it is necessary to confirm that the apparent leg discomfort occurs or worsens with rest and improves with activity.

RLS affects the awake individual but causes tremendous nocturnal sleep disturbance. RLS delays sleep onset because the affected individual has to keep moving the legs about to satisfy the urge to move and to keep unpleasant sensations in the legs at bay. $0 \mathrm{ften}$, RLS sufferers report sleep latency of an hour or longer after getting in bed for the night. Sleep tends to occur only when overwhelming sleepiness sets in late in the night. H owever, this sleep tends to be shallow and easily disrupted. RLS sufferers often have periodic leg movements in sleep (described in detail below) that usually cause brain micro-arousals but may cause completeawakening too. RLS tends to recur during such awakenings, causing great difficulty getting back to sleep. The consequent sleep deficit is often difficult to make up and the person feels un-refreshed by night sleep and is sleepy during the day. RLS sufferers describe poor quality of life and sub-normal energy levels. RLS may contribute to affective disorders.

Physical examination is unrevealing in RLS except for possible observable periodic leg movements while awake or thetypical rhythmic movement of thelegs while seated that patients may display. Physical examination in secondary RLS may be noteworthy for stigmata of the underlying medical problem.

\section{Periodic Leg Movements}

Periodic leg movements may occur in sleep or during wakeful rest. Periodic leg movements are evenly spaced out repetitive movements of the legs. Periodic upper extremity activity in sleep is seen relatively infrequently.

Periodic leg movements in wakefulness (PLM W) are involuntary but controllable movements of the legs that mostly occur later in the day and may be directly observable. The occurrence of over 40 PLMW during one hour of wakeful rest correlates with RLS.

Periodic leg movements in sleep (PLMS) are diagnosed on polysomnography. The patient's bed partner may describe repeated jerking movements of the legs occurring while the patient is asleep. Sometimes bed partners report being disturbed or even injured by such movements. Patients themselves may confirm that sheets they cover themselves with in bed are very disarrayed at the foot end by morning.

The specific movements in PLM S may vary from person to person. Detailed analysis of the sequence of movements, classically described as having multiple components starting with dorsiflexion of the hallux, then dorsiflexion at the ankle and then possibly flexion at the knee and hip, is not needed for routine diagnostic purposes. Bilateral electro-myographic recording from the tibialis anterior as part of a polysomnogram is sufficient. PLMS mainly occur in non-REM sleep. O ccurrence of PLMS in REM sleep may be facilitated by use of medications such as selective serotonin reuptake inhibitor (SSRI) anti-depressants that prevent the usual degree of muscle tone decline in sleep. O ne limb (or both together) may exhibit PLM S consistently or there may be variation related to posture. The defining characteristics of PLM S are described in the section on tests. PLM S is often associated with brain micro-arousal. This results in sleep fragmentation, the feeling of unrefreshing sleep and excessive daytime sleepiness. 


\section{Differential diagnosis}

RLS is differentiated from akathisia by the classic tetrad of RLS including uncomfortable sensations in the extremities, urge to move the extremities, relief of the unpleasant uncomfortable sensations by movement and circadian variation of symptoms. Akathisia is involuntary motor activity alone without the features of RLS. D opaminergic antagonists may cause irreversible akathisia. The dysesthesia of peripheral neuropathy is usually constant and not associated with theurge to move the limbs, relief of sensations by movement or circadian variation. Similarly, lower extremity arterial insufficiency can cause rest pain but such discomfort usually worsens on exertion and doesn't have a circadian pattern. Parkinsonism is not accompanied by the RLS diagnostic tetrad.

Sleep starts at sleep onset are involuntary movements that involve the whole body and don't have periodicity. N octurnal leg cramps also do not show the periodicity typical of PLMS. Respiratory-effort-related leg movements in sleep (RERLM S) occur towards the ends of upper airway resistance syndrome (UARS) cycles of crescendo breathing or at the end of overt airflow obstruction events. RERLM S can sometimes occur at very regular intervals but occurrence of the leg movements only at the end of sleep-disordered breathing episodes distinguishes RRLM S from PLM S. PLM S may worsen as O SA is corrected using positive ai rway pressure therapy in sleep while RERLM S are eliminated. SSRI and tri-cyclic anti-depressants may prevent muscle tone decline in sleep and may also cause repeated fasciculation of muscles that is difficult to distinguish from PLM S the absence of distinct movements on the leg electromyograms may help distinguish muscle fasciculation. Phasic REM sleep may be associated with brief isolated limb movements without periodicity. Seizures in sleep are not usually limited to just the legs and any shaking or twitching of the extremities during a seizure tends to be far faster than the typical frequency of PLMS.

\section{MANAGEMENT}

\section{Testing}

Every patient diagnosed with RLS and/or PLM S should be evaluated for possible secondary causes. Blood tests should include a complete blood count (including red cell indices), iron, ferritin, vitamin B12, folic acid, blood urea nitrogen and creatinine levels, as well as fasting and post-prandial ( 2 hours after consumption of a 75 gram rapidly absorbed carbohydrate drink) blood glucose levels. Any abnormalities should be investigated further keeping age and local disease prevalences in mind and treated appropriately. It is this author's observation that pre-diabetes is often newly identified during such evaluation of middle-aged and older patients with SRLMD.

The Suggested Immobilization Test (SIT) [30, 31] may be needed if there is substantial doubt about the diagnosis of RLS but is not done routinely to diagnose RLS in most sleep disorders centers. The SIT is performed about 1 hour prior to the patient's usual bedtime, with the patient asked to sit still in a bed reclined back at 45 degrees with eyes open and with the legs outstretched for a period of 1 hour. The patient quantifies the perceived level of leg discomfort every five minutes on a $100 \mathrm{~mm}$ visual analog scale based on which an average leg discomfort score is computed. A score of greater than 11 is moderately correlated with RLS. Electromyographic monitoring of the lower extremities is maintained for the entire hour of the SIT. PLM W, which may last longer than PLM S, are scored if they last 0.5 to 10 seconds and occur in sequences of 4 or more movements with inter-movement intervals of 4 to 90 seconds.

On overnight polysomnography, long sleep latency with frequent leg electromyogram activity during wakefulness and videographic evidence of typical leg movements on rapidly scrolling through the video file may sometimes be needed to clinch the diagnosis of RLS. In contrast to RLS that is mainly diagnosed clinically, PLM $S$ is diagnosed on polysomnography. PLM $S$ usually occur 20 to 40 seconds apart but may occur from 4 to 90 seconds apart. PLM S may last 0.5 to 5 seconds each. A sequence of at least 4 evenly spaced movements within the above timeframe is needed to qualify as PLM S. The amplitude of PLM S on the EM G should be at least $25 \%$ of the amplitude obtained with foot dorsiflexion during bio-calibrations at the start of the study. The number of PLM S per hour of sleep (the PLM S Index), is used to assess severity of PLMS. A PLM S index less than 5 is considered normal. A PLM S index between 5 and 25 is considered mild. M oderately severe PLM $S$ is defined as a PLM S Index between 25 and 50 and PLM S is considered severe if the PLM S Index is greater than 50 . PLM S occur in around $80 \%$ of patient with RLS. If the 
PLM S Index less is than 5 times per hour of sleep, RLS is unlikely. Averaging for night to night variation in PLM S, a PLM S index greater than 11 times per hour of sleep on either of two consecutive nights of polysomnography strongly supports the diagnosis of RLS.

The M ultiple Sleep Latency Test (M SLT) should not be performed until all of an individual's causes of nocturnal sleep disruption have been effectively treated on an ongoing basis.

\section{Therapy}

In the last few years, along with advances in the understanding of the pathophysiology of RLS, consensus has developed regarding effective, well-tolerated therapy for SRLM D. M edication to control SRLM D should be initiated if symptoms are frequent (more than $2-3$ times a week), bothersome, worsening, disrupting night sleep or causing excessive daytime sleepiness. If SR LM D severely disrupt night sleep in a patient also suspected to have sleep-disordered breathing (SDB), it may be necessary to first control SRLM D and then evaluate the patient for SD B, though it is preferable to treat SD B first as it allows RRLM $S$ to be distinguished from PLM S.

Along with initiation of medical therapy for SRLM D, potential offending agents such as caffeine should be withdrawn [32]. To the extent possible, patients on antidepressants should be switched to anti-depressants with relatively low sleep-related side-effect profiles such as citalopram or escitalopram.

Any identified secondary causes of SRLM D should be treated appropriately though it is often reasonable to simultaneously begin specific therapy such as dopamine receptor agonists to control SRLMD. O nce the underlying medical problem is adequately controlled, withdrawal of dopaminergic medication can be attempted. Blood donors should be instructed to stop donating blood.

D opamine receptor agonists are highly effective in controlling SRLM D in small doses and are the mainstay of therapy now. The two dopaminergic agonists currently used to the greatest extent for these conditions are the non-ergot-derived agents pramipexoleand ropinirole[33, 34, 35]. Pramipexole $0.125 \mathrm{mg}$ to $0.25 \mathrm{mg} \mathrm{H} \mathrm{S}$ controls RLS symptoms starting close to bedtime and PLM S. An earlier dose may be needed in the afternoon or evening to control RLS symptoms occurring earlier. A morning dose is needed rarely. The total daily dose of pramipexole needed to control SRLM D is usually far less than $1 \mathrm{mg}$. Equipotent doses of ropinirole tend to be slightly higher than those of pramipexole (typically starting at $0.25 \mathrm{mg}$ - $0.5 \mathrm{mg} \mathrm{H} \mathrm{S}$ ). Ropinirole is not excreted renally and so is preferred in patients with renal dysfunction. Most patients experiencedramatic benefit, with control of RLS, improvement of sleep maintenance, better sleep quality and less daytime sleepiness, within a few doses itself. A few patients can't tolerate these dopaminergic agonists despite the low doses used and experience agitation or jitteriness. Even less frequently, patients report either excessive sleepiness or difficulty initiating sleep at the desired bedtime despite control of SRLM D. W ithdrawal of medication is rapidly effective in reversing such symptoms. Ergot-derived dopamine receptor agonists such as pergolide and cabergoline (and even bromocriptine per a case report) may be associated with development of serious multi-valvular heart disease in a few patients [36] and so would be much less preferred.

Levodopa, in combination with carbidopa or benserazide to inhibit peripheral conversion of L-D OPA to dopamine, is second line therapy for SRLM D. A bedtime dose of 1 to 2 tablets of levodopa / carbidopa 100/25 mg can control latenight RLS and PLM S. A similar dose or two may be needed for RLS occurring earlier in the day. Levodopa / carbidopa therapy can lead to augmentation and rebound of symptoms. Augmentation is the occurrence of RLS symptoms earlier in the day compared with the onset timebeforeinitiation of therapy and is much less of an issue with pramipexole and ropinirole [37]. Augmentation and other side-effects don't recur or cross-over if a patient is switched from levodopa to a dopamine agonist or from one dopamine agonist to another.

Benzodiazepines, primarily clonazepam, may reduce brain arousal caused by periodic leg movements in sleep and shorten sleep latency. Clonazepam 0.25 to $0.5 \mathrm{mg}$ HS is sufficient for most patients and excessive dosing should be avoided as it's likely to cause excessive sleepiness the next day. Clonazepam can be used along with dopamine agonists or levodopa.

0 piates may help control pain that may be a significant component of symptoms of some patients with SRLMD, especially if they also have neuropathy. Addiction potential of opiates limits their use.

$G$ abapentin has been reported, in relatively small studies, to improve both sensory symptoms and motor 
activity associated with RLS, the severity of PLM $S$ and sleep architecture [38]. Relatively high doses of gabapentin (around $1800 \mathrm{mg} /$ day total) are needed for benefit. Gabapentin can be tried if the patient cannot tolerate any dopaminergic medication.

\section{CONCLUSION}

RLS and PLM S are fairly common sleep disorders that have significant adverse consequences. The true prevalence of SRLMD in different population groups around the globeneeds to be determined by appropriately sized cross-sectional studies. Various secondary causes have been identified that seem to manifest as SRLM D through the final common pathway of dopaminergic dysfunction in the central nervous system. The exact genetic basis and molecular mechanism of familial and primary RLS is yet to be determined. Effective, readily available and well-tolerated therapies for SRLM D have been established.

\section{Authors' affiliations}

Samavedam A. Krishna, M. D., Diplomate, American Boards of Internal Medicine and Sleep Medicine, Mount Vernon, O hio, USA

\section{References}

1. Ekbom K. Restless legs: a clinical study. Acta Med Scand Suppl 1945; 158.

2. Allen RP, Picchietti $D$, Hening WA, Trenkwalder $C$, Walters AS, Montplaisir J. Restless legs syndrome: diagnostic criteria, special considerations and epidemiology. A report from the restless legs syndrome diagnosis and epidemiology workshop at the National Institutes of Health. Sleep Med 2003; 4: $101-19$.

3. In Hauri P, Sateia MJ. The international classification of sleep disorders, $2^{\text {nd }}$ Edition. Westchester, IL: American Academy of Sleep Med, 2005. p. 178 - 86.

4. Tison F, Crochard A, Leger D, Bouee S, Lainey E, El Hasnaou A. Epidemiology of restless legs syndrome in French adults. A nationwide survey: The IN STANT Study. N eurology 2005; 65: $239-46$

5. Ulfberg J, Nystrom B, Carter N, Edling C. Prevalence of restless legs syndrome among men aged 18 to 64 years: an association with somatic disease and neuropsychiatric symptoms. Mov Disord 200; 16 (6): 1159 - 63.

6. Lavigne GJ, Montplaisir JY. Restless legs syndrome and sleep bruxism: prevalence and association among Canadians. Sleep 1994; 17: 739 - 43.

7. Hening W, Walters AS, Allen RP, Montplaisir J, Myers A,
Ferini-Strambi L. Impact, diagnosis and treatment of restless legs syndrome in a primary care population: the REST (RLS epidemiology, symptoms and treatment) primary care study. Sleep Med 2004; 5: 237 - 46.

8. Ondo WG, Vuong KD, Wang $Q$. Restless legs syndrome in monozygotic twins: clinical correlates. Neurology 2000; 55 (9): $1404-6$

9. Allen RP, La Buda MC, Becker P, Earley CJ. Family history study of the restless legs syndrome. Sleep Med 2002; 3: $53-7$.

10. Montplaisir J, Boucher S, Poirier G, Lavigne G, Lapierre 0 , Lesperance P. Clinical, polysomnographic and genetic characteristics of restless legs syndrome: a study of 133 patients diagnosed with new standard criteria. Mov Disord 1997: 12: 61 - 5

11. Winkelmann J. The genetics of restless legs syndrome. Sleep Med 2002; 3: S9 - S12.

12. Ulfberg J, Nystrom B. Restless legs syndrome in blood donors. Sleep Med 2004; 5: 115 - 8 .

13. Rothdach AJ, Trenkwalder C, Haberstock J et al. Iron metabolism and the risk of restless legs syndrome in an elderly population: The MEMO Study. J N eurol 2002; 249: 1195 - 9.

14. Unruh ML, Levey S, D'Ambrosio C, Fink NE, Powe NR, Meyer KB. Restless legs symptoms among incident dialysis patients: association with lower quality of life and shorter survival. Am J Kidney Dis 2004; 43 (5): 900 - 9.

15. Bhowmik D, Bhatia M, Gupta S, Agarwal SK, Tiwari SC, Dash SC. Restless legs syndrome in hemodialysis patients in India: a case controlled study. Sleep Med 2003; 4: 143 -6 .

16. Krishnan PR, Bhatia M, Behari M. Restless legs syndrome in Parkinson's Disease: a case-controlled study. M ov Disord 2003; 18: 181 - 5.

17. Tan EK, Seah A, See SJ, Lim E, Wong MC, Koh KK. Restless legs syndrome in an Asian population: a study in Singapore. Mov Disord 2001; 16 (3): 577 - 9.

18. Mizuno S, Miyaoka T, Inagaki T, Horiguchi J. Prevalence of restless legs syndrome in non-institutionalized Japanese elderly. Psychiatry Clin Neurosci 2005; 59: 461 - 5.

19. Michaud M, Soucy J, Chabli A, Lavigne G, Montplaisir J. SPECT imaging of striatal pre- and postsynaptic dopaminergic status in restless legs syndrome with periodic leg movements in sleep. J Neurol 2002; 249: $164-70$.

20. Karlsson P, Farde L, Halldin C. Circadian rhythm in central D1-like dopamine receptors examined by PET. (Special issue: abstracts from Third International Symposium on functional neuroreceptor mapping (New York, USA)) J Neuroimaging 2000

21. Sloand JA, Shelly MA, Feigin A, Bernstein P, Monk RD. A double-blind, placebo-controlled trial of intravenous iron dextran therapy in patients with ESRD and restless legs syndrome. Am J Kidney Dis 2004; 43 (4): 663 - 70.

22. Earley CJ, Heckler D, Allen RP. The treatment of restless legs syndrome with intravenous iron dextran. Sleep Med 2004: 5: 231 - 35 .

Indian Journal of Sleep M edicine (IJSM ), Vol. 1, N o. 1, 2006 
23. Barton JC, Wooten VD, Acton RT. Hemochromatosis and iron therapy of restless legs syndrome. Sleep Med 2001; 2: $249-51$

24. Allen RP, Barker PB, Wehrl F, et al. MRI measurement of brain iron in patients with restless legs syndrome. N eurology 2001; 56 (2): $263-5$.

25. Earley CJ, Connor JR, Beard JL, Malecki EA, Epstein DK, Allen RP. Abnormalities in CSF concentrations of ferritin and transferrin in restless legs syndrome. Neurology 2000 54: $1698-1700$.

26. Skomro RP, Ludwig S, Salamon E, Kryger MH. Sleep complaints and restless legs syndrome in adult type 2 diabetics. Sleep Med 2001; 2: 417 - 22.

27. Happe S, Treptau N, Ziegler R, Harms E. Restless legs syndrome and sleep problems in children and adolescents with insulin-dependent diabetes mellitus type 1 . Neuropediatrics 2005; 36: 98 - 103.

28. Wang GJ, Volkow ND, Logan J, et al. Brain dopamine and obesity. Lancet. 2001; 357(9253): 354 - 7.

29. Bottiglieri T, Laundy M, Crellin R, Toone BK, Carney MWP, Reynolds $\mathrm{EH}$. Homocysteine, folate, methylation and monoamine metabolism in depression. J. Neurol N eurosurg Psychiatry 2000; 69: 228 - 32.

30. Montplaisir J, Boucher S, Nicholas A, et al. Immobilization tests and periodic leg movements in sleep for the diagnosis of restless legs syndrome. Mov Disord 1998; 13: 324 - 9

31. Michaud M, Poirier G, Lavigne G, Montplaisir J. Restless legs syndrome: scoring criteria for leg movements recorded during the suggested immobilization test. Sleep Med 2001; 2: $317-21$.

32. Lutz EG. Restless legs, anxiety and caffeinism. J Clin Psychiatry. 1978; 39 (9): 693 - 8.

33. Montplaisir J, Nicolas A, Denesle R, Gomez-Mancilla B. Restless legs syndrome improved by pramipexole: a doubleblind randomized trial. Neurology 1999; 52 (5): 938 43.

34. Manconi M, Casetta I, Govoni V, Cesnik E, Ferini-Strambi L, Granieri E. Pramipexole in restless legs syndrome evaluation by suggested immobilization test. J N eurol 2003; 250: 1494 - 5 .

35. Walters AS, O ndo WG, Dreykluft $T$, Grunstein R, Lee $D$, Sethi K. Ropinirole is effective in the treatment of restless legs syndrome. TREAT RLS 2: A 12-week, double-blind randomized, parallel-group, placebo-controlled study. M ov Disord 2004; 19 (12): 1414 - 23

36. Horvath J, Fross RD, Kleiner-Fisman G, et al. Severe multivalvular heart disease: a new complication of the ergot derivative dopamine agonists. Mov Disord 2004; 19 (6): $656-62$.

37. Ondo W, Romanyshyn J, Vuong KD, Lai D. Long-term treatment of restless legs syndrome with dopamine agonists. Arch Neurol. 2004; 61(9):1393 - 7.

38. Garcia-Borreguero D, Larrosa O, de la Llave $Y$, Verger K, Masramon X, Hernandez G. Treatment of restless legs syndrome with gabapentin: a double-blind, cross-over study. Neurology. 2002; 59 (10): 1573 - 9. 